\title{
Performance and uncertainty analysis in machine-assisted design of photonic devices
}

\author{
Daniele Melati*,a, Yuri Grinberg ${ }^{\mathrm{b}}$, Abi Waqas ${ }^{\mathrm{c}}$, Paolo Manfredid, Mohsen Kamandar Dezfouli ${ }^{\mathrm{a}}$, \\ Pavel Cheben ${ }^{\mathrm{a}}$, Jens H. Schmid ${ }^{\mathrm{a}}$, Siegfried Janz ${ }^{\mathrm{a}}$, Andrea Melloni ${ }^{\mathrm{e}}$, and Dan-Xia Xu ${ }^{\mathrm{a}}$ \\ ${ }^{a}$ Advanced Electronics and Photonics Research Center, National Research Council Canada, 1200 \\ Montreal Rd., Ottawa, ON K1A 0R6, Canada; ${ }^{b}$ Digital Technologies Research Center, National \\ Research Council Canada, 1200 Montreal Rd., Ottawa, ON K1A 0R6, Canada; ${ }^{\circ}$ Departement of \\ Telecommunication Engineering, Mehran University of Engineering and Technology, Jamshoro, \\ 76062 Sindh, Pakistan; ${ }^{\mathrm{d} D e p a r t m e n t}$ of Electronics and Telecommunications, Politecnico di Torino, \\ 10129 Torino, Italy; 'Dipartimento di Elettronica, Informazione e Bioingegneria, Politecnico di \\ Milano, via Ponzio 34/5, 20133 Milano, Italy.
}

\begin{abstract}
Machine-assisted design of integrated photonic devices (e.g. through optimization and inverse design methods) is opening the possibility of exploring very large design spaces, novel functionalities and non-intuitive geometries. These methods are generally used to optimize performance figures-of-merit. On the other hand, the effect of manufacturing variability remains a fundamental challenge since small fabrication errors can have a significant impact on light propagation, especially in high-index-contrast platforms. Brute-force analysis of these variabilities during the main optimization process can become prohibitive, since a large number of simulations would be required. To this purpose, efficient stochastic techniques integrated in the design cycle allow to quickly assess the performance robustness and the expected fabrication yield of each tentative device generated by the optimization. In this invited talk we present an overview of the recent advances in the implementation of stochastic techniques in photonics, focusing in particular on stochastic spectral methods that have been regarded as a promising alternative to the classical Monte Carlo method. Polynomial chaos expansion techniques generate so called surrogate models by means of an orthogonal set of polynomials to efficiently represent the dependence of a function to statistical variabilities. They achieve a considerable reduction of the simulation time compared to Monte Carlo, at least for mid-scale problems, making feasible the incorporation of tolerance analysis and yield optimization within the photonic design flow.
\end{abstract}

Keywords: photonic devices, silicon photonics, probability theory, stochastic processes, machine learning, pattern recognition, principal component analysis, uncertainty analysis

\section{INTRODUCTION}

Integrated photonic components are steadily making their way into modern communication networks and also have growing applications in computing, automation, sensing and life sciences. At the same time, novel photonic devices that employ increasingly complex geometries and metamaterials are continuously proposed $[1,2]$. In these scenarios, the number of design parameters vastly increase and manual device design is often impractical. Machine-assisted design exploiting tools such as the genetic algorithm, particle swarm, and gradient-based optimization are increasingly used to search more efficiently for high-performance designs [3]. Inverse design methods have been proposed to tackle this challenges, demonstrating highly compact devices employing non-intuitive structures [4]. Supervised machine learning methods such as the artificial neural network have been used to speed up the search and optimization process [5]. Recently, we have proposed the use of a suite of machine learning tools, including global optimization, supervised learning and unsupervised machine learning pattern recognition, to create a global map of a multi-parameter design spaces while using a tractable amount of time and computational resources [6].

Despite this rapid evolution in the design methodology, the analysis of the impact of manufacturing variability remains a major challenge [7]. Uncertainty due to fabrication often limits the sustainable complexity and poses major problems in

*daniele.melati@nrc-cnrc.gc.ca 
achieving high production yield [8]. This is particularly true for high-index-contrast technologies where small fabrication deviations in the waveguide geometry and circuit topology have significant impacts on light propagation.

The possibility to exploit the information on variability within machine-assisted design approaches would allow both predicting its effects on the final designs and also optimizing layouts to maximize stochastic quantities such as fabrication yield. Unfortunately, classical methods for stochastic analysis such as Monte Carlo require a very large number of simulations to obtain reliable results, hampering their application into large-scale multi-parameter designs. Stochastic spectral methods have been regarded as a promising and efficient alternative to Monte Carlo for statistical analysis also in photonics $[9,10]$. Polynomial chaos expansion (PCE) techniques allow to build a surrogate model of an unknown random function by representing its dependence on stochastic variables by means of an orthogonal set of polynomials. Accurate and efficient variability representation has been demonstrated for several photonic devices, also in the case of correlated random variables [11,12]. Models based on generalized polynomial chaos expansion have been recently exploited for circuit design optimization under fabrication uncertainty $[13,14]$. These techniques can provide a viable way to introduce uncertainty information at into machine-assisted design flows.

In this invited talk we report on the application of polynomial chaos expansion for the stochastic analysis of photonics devices and circuits. We first present the analysis of a compact $(15 \mu \mathrm{m})$ vertically emitting grating coupler with a single random variable using both classical PCE and a combination of PCE and frequency-domain macromodeling (vector fitting). The latter enables a very efficient investigation of the stochastic properties of frequency-dependent transfer functions. We then show how PCE can be used to handle also the case of multiple correlated random variables. We perform the analysis of a 4.5-mm-long second-order unbalanced Mach-Zehnder filter with five random variables exploiting the Karhunen-Loeve (KL) transform.

\section{SURROGATE MODELS WITH POLYNOMIAL CHAOS EXPANSION}

In order to investigate the effect of uncertainty, we define the transfer function of a photonic device or circuit as $\mathrm{Y}(\mathrm{f}, \vec{\xi})$. $\mathrm{Y}$ depends in general on frequency $\mathrm{f}$ and on a vector of $\mathrm{N}$ independent random variables $\overrightarrow{\boldsymbol{\xi}}=\left[\xi_{1}, \xi_{2}, \ldots, \xi_{\mathrm{N}}\right]$. Commonly $\mathrm{Y}$ is not available in an analytical form and its computation can be lengthy, making the repeated simulations required by Monte-Carlo-type analyses to estimate stochastic moments (e.g. mean, variance, probability density function...) impractical. This problem can be particularly emphasized when the computation of the moments is required as part of an optimization routine because thousands of simulations would be required at each step of the optimization that could require itself several thousands of iterations [14].

A possible way to overcome this limitation is defining an analytical surrogate model describing the dependence of $Y$ on $\vec{\xi}$ [15]. If the computation of this approximate model is done efficiently, the availability of an analytical expression for Y could then make the computation of its stochastic moments inexpensive. To this purpose, within the polynomial chaos expansion (PCE) formalism, the dependence of $\mathrm{Y}$ on $\vec{\xi}$ is described as a summation of basis functions $\varphi_{\mathrm{i}}(\vec{\xi})$ with suitable coefficients $\mathrm{y}_{\mathrm{i}}(\mathrm{f})$ as [15]

$$
\mathrm{Y}(\mathrm{f}, \vec{\xi})=\sum_{\mathrm{i}=0}^{\infty} \mathrm{y}_{\mathrm{i}}(\mathrm{f}) \varphi_{\mathrm{i}}(\vec{\xi})
$$

In this expression, the coefficient $y_{i}$ depends in general on frequency and $\varphi_{i}$ are orthonormal polynomials with respect to the probability measure $\mathrm{W}(\vec{\xi})$

$$
\left\langle\varphi_{\mathrm{i}}(\vec{\xi}), \varphi_{\mathrm{j}}(\vec{\xi})\right\rangle=\int \varphi_{\mathrm{i}}(\vec{\xi}) \varphi_{\mathrm{j}}(\vec{\xi}) \mathrm{W}(\vec{\xi}) \mathrm{d} \vec{\xi}=\delta_{\mathrm{ij}}
$$

where $\delta_{\mathrm{ij}}$ is the Kronecker delta. If the random variables $\vec{\xi}$ are independent, the corresponding basis functions $\varphi_{\mathrm{i}}(\vec{\xi})$ can be computed as the product of the orthogonal polynomials corresponding to each individual random variable $\xi_{\mathrm{i}}$. For common probability density functions (i.e., Gaussian, Uniform...), the basis functions are readily available and described by the Wiener-Askey scheme [15]. For example for Gaussian distributed variables the basis functions are the Hermite polynomials. 
For computational purposes the PCE model (1), involving an infinite series, must be truncated. The truncation is done considering all $\mathrm{N}$-dimensional polynomials up to the order $\mathrm{P}$ (that is chosen depending on the problem), with a total number of elements (basis functions) $M$ defined as

$$
\mathrm{M}+1=\frac{(\mathrm{N}+\mathrm{P}) !}{\mathrm{N} ! \mathrm{P} !}
$$

The last step is the computation of the coefficients $\mathrm{y}_{\mathrm{i}}$. In this work this is done generating a small pool of $\mathrm{K}$ realizations for the random variables $\vec{\xi}$, indicated as $\left[\vec{\xi}_{\mathrm{j}}\right]_{1}^{\mathrm{K}}$. For each realization, the transfer function $\mathrm{Y}$ is simulated and used to build the linear system

$$
\Psi \mathbf{y}=\mathbf{R}
$$

where $\mathbf{y}$ is the vector of the $\mathrm{M}$ coefficients $\mathrm{y}_{\mathrm{i}}$, the $\mathrm{j}^{\text {th }}$ row of the $[\mathrm{KxM}]$ matrix $\boldsymbol{\Psi}$ contains the multivariate polynomial basis evaluated at $\vec{\xi}_{\mathbf{j}}$ and the vector $\mathbf{R}$ contains the results of the K simulations. The (normally undetermined) system (4) is eventually inverted to compute the coefficients $\mathrm{y}_{\mathrm{i}}$. When this is done using a least-square solver, $\mathrm{K}=2 \mathrm{M}$ commonly ensures both a reliable solution and a number of initial simulations $\mathrm{K}$ orders of magnitude smaller than that required by Monte-Carlo approach. K can be reduced even more exploiting compressed sensing techniques [13].

Since in general the coefficients $y_{i}$ depends on frequency, this process must be repeated for each frequency point. Alternatively, PCE can be combined with frequency-domain macromodeling such as the vector-fitting technique to further improve its efficiency [16]. In this case the vector-fitting algorithm is first applied individually to the $\mathrm{K}$ initial simulations, obtaining a set of frequency-independent matrices that depend only on the random variables $\vec{\xi}$. PCE is then applied on these matrices once and finally the frequency-dependent coefficients $y_{i}$ are retrieved through the vector-fitting model.

Once available, model (1) efficiently represents the system response to variability and allows computing the stochastic moments of $Y$ with minimal resources and time. Mean $\mu$ and standard deviation $\sigma^{2}$ can be analytically computed as

$$
\begin{gathered}
\mu=y_{0}, \\
\sigma^{2}=\sum_{1}^{M} y_{i}^{2}\left\langle\varphi_{i}(\vec{\xi}), \varphi_{i}(\vec{\xi})\right\rangle .
\end{gathered}
$$

The probability density function (PDF) and the cumulative density function (CDF) can be computed inexpensively for example applying Monte Carlo sampling on the analytical PCE approximation (1).

\section{MACHINE-ASSISTED DESIGN AND STOCHASTIC ANALYSIS OF PHOTONIC DEVICES}

As a design study case we consider here a vertically-emitting grating coupler in silicon-on insulator technology [17]. As shown in Fig. 1(a) the grating period consists of a pillar of $220 \mathrm{~nm}$ in height and an L-shaped section partially etched to $110 \mathrm{~nm}$. The blazing effect generated by the former section ensures that light is primarily diffracted upwards. The structure dimensions $\mathrm{L}_{1}-\mathrm{L}_{5}$ define the five-dimensional design parameter space. The optimization objective is the coupling efficiency CE of the diffracted TE-polarized light to a standard single mode optical fiber (SMF-28) placed vertically on top of the grating.

Recently, we have proposed a novel machine-learning-based design methodology that instead of resulting in a single optimized final device allows to generate a global map of the multi-parameter design space, providing the designer insights on behavioral patterns and enabling informed decisions based on different figures-of-merits [6]. Global optimization combined with a Fourier-type 2-D eigenmode expansion simulator [18] is first used to search for a small set of designs with state-of-the-art fiber coupling efficiency, resulting in a pool of 45 good grating designs with CE $>0.74$. Principal component analysis (PCA) is then used to search for patterns within this pool in the form of linear approximations to the dataset. Two principal components are found to be sufficient to accurately represent 

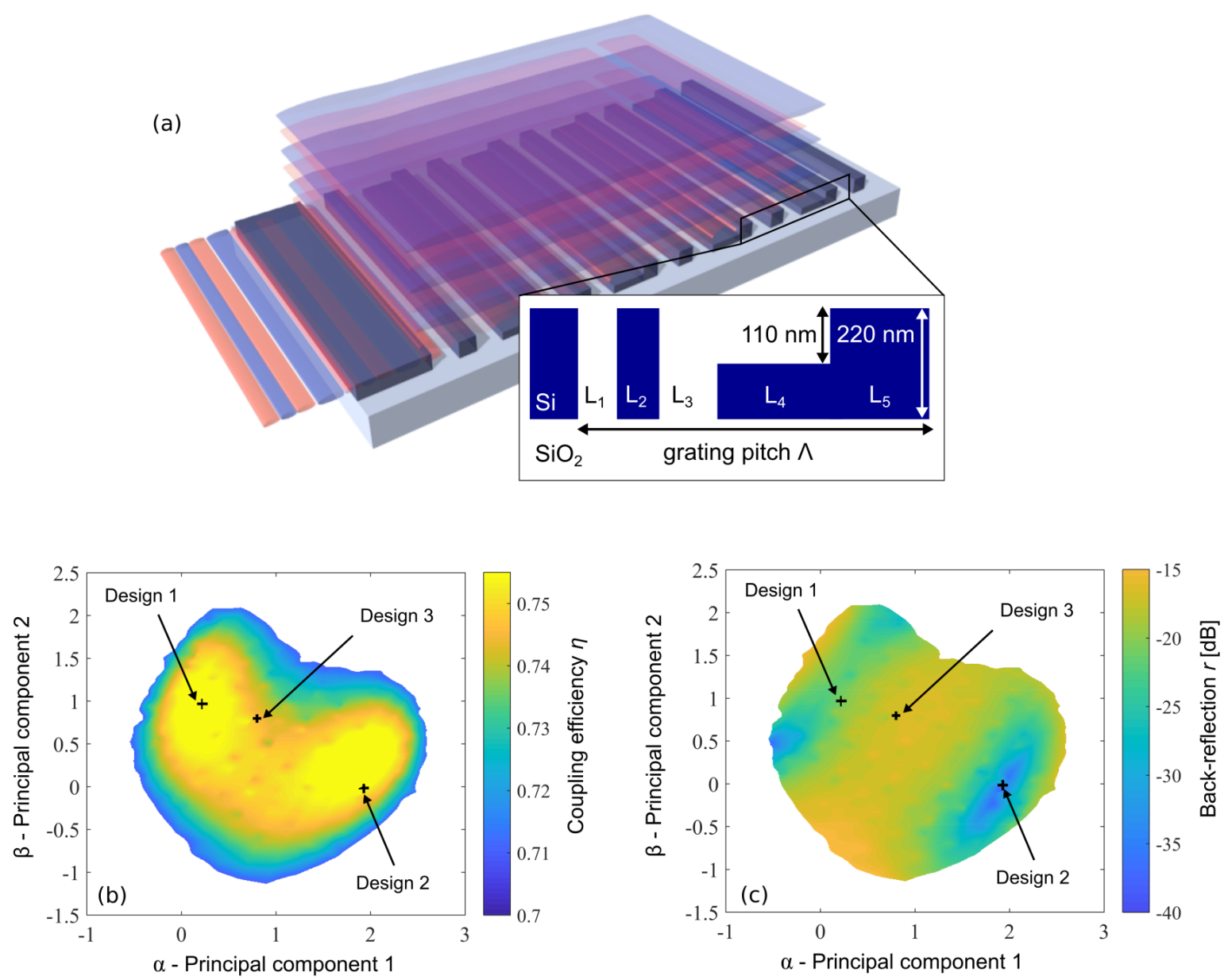

Figure 1: (a) Schematic of the vertical grating coupler. The design involves five parameters (the length of each section $\mathrm{L}_{1}$ - $\mathrm{L}_{5}$ ).

Using a combination of machine learning techniques, including pattern recognition, the 5-D design space can be efficiently explored, identifying a 2-D sub-space (hyperplane) containing a large number of designs with state-of-the-art coupling efficiency (b) and back-reflection (c). Only devices with coupling efficiency larger than 0.7 are shown.

Table 1. Properties of the three grating designs shown in Fig. 1. Despite different design parameters, the three designs have all coupling efficiency above $74 \%$ and back-reflection smaller than $-20 \mathrm{~dB}$ at $\lambda=1550 \mathrm{~nm}$.

\begin{tabular}{|c|c|c|c|c|c|c|c|c|}
\hline Design & L1 & L2 & L3 & L4 & L5 & $\begin{array}{c}\text { Period } \\
{[\mathbf{n m}]}\end{array}$ & CE & $\mathbf{R}[\mathbf{d B}]$ \\
\hline 1 & 77 & 84 & 115 & 249 & 171 & 696 & 0.77 & -21 \\
\hline 2 & 102 & 80 & 117 & 330 & 98 & 727 & 0.78 & -44 \\
\hline 3 & 81 & 85 & 109 & 276 & 149 & 700 & 0.75 & -24 \\
\hline
\end{tabular}

the entire pool. That is, all good designs approximately lie on a 2-D hyperplane - the reduced parameter space - and the rest of the design space can be excluded from further investigation. Since the number of parameters has been reduced from the original five $\left(\mathrm{L}_{1}-\mathrm{L}_{5}\right)$ to the two coefficients of the principal components $(\alpha$ and $\beta)$, the exhaustive exploration of the design space is now feasible. Figure 1(b) report a global mapping of the coupling efficiency within this design space for $\lambda=1550 \mathrm{~nm}$. Each point $(\alpha, \beta)$ represents a specific set $\mathrm{L}_{1}-\mathrm{L}_{5}$. Figure 1(c) shows the mapping for the back- 

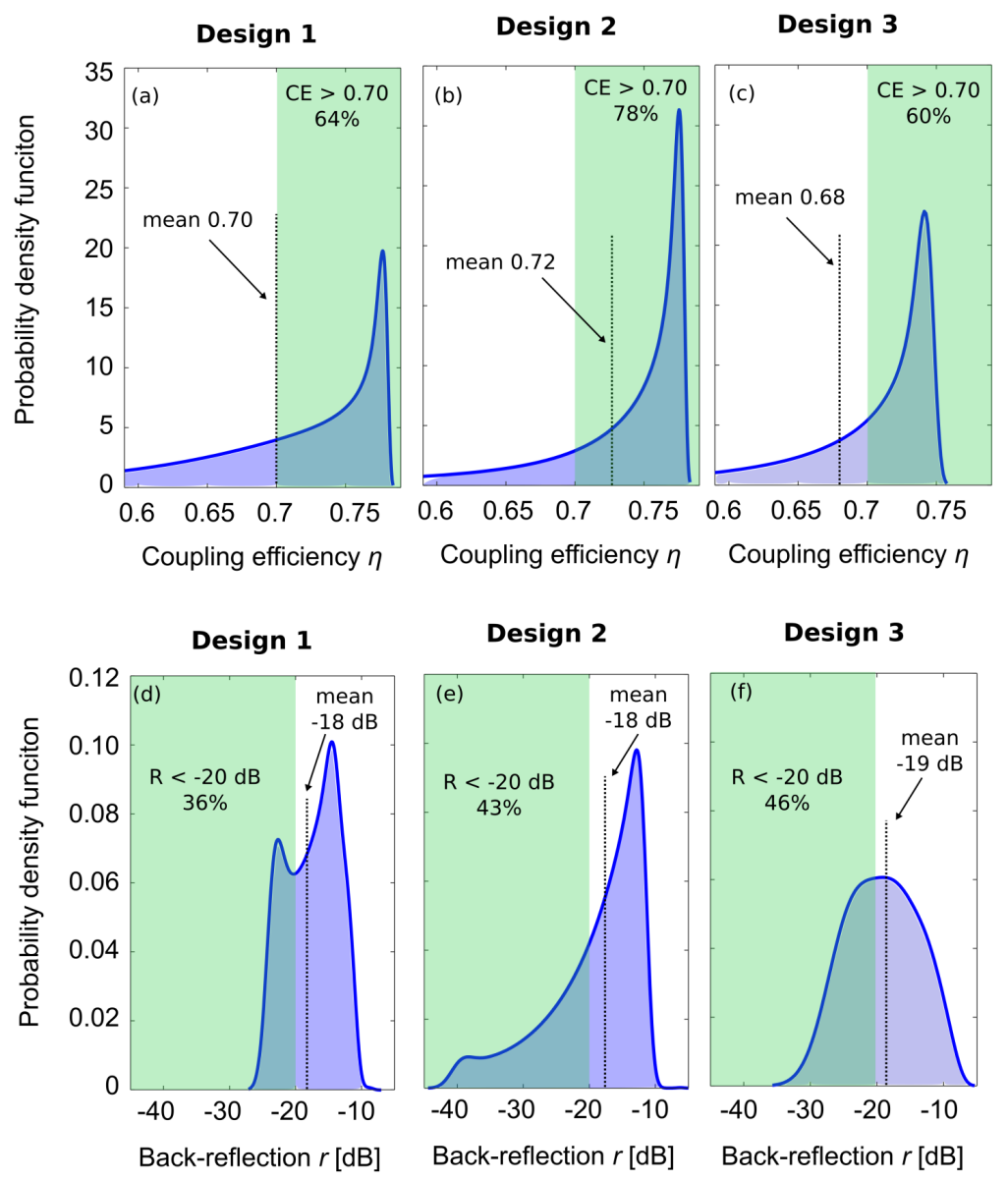

Design 3

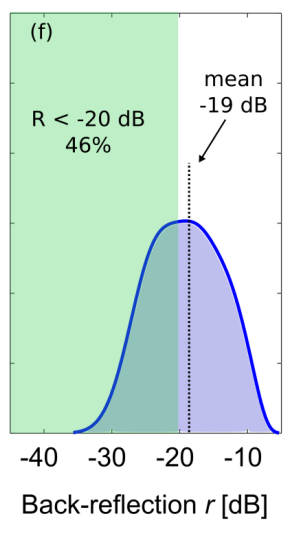

Figure 2: Probability density function of the coupling efficiency (a)-(c) and back-reflection at $\lambda=1550 \mathrm{~nm}$ for the three designs described in Fig. 1 and Table 1. Average values are marked with dotted lines. Green areas highlight specification requirements: coupling efficiency larger than 0.7 and back-reflection smaller than $-20 \mathrm{~dB}$.

reflections generated by the grating in the waveguide at $\lambda=1550 \mathrm{~nm}$. Back-reflections were not included in the design procedure but are evaluated as an additional performance metric only for the designs included in the reduced parameter space. As an example three possible designs are identified on the maps. For each design, Tab. 1 reports the length of each section, the period, the coupling efficiency CE and back-reflections R. All designs are about 15- $\mu \mathrm{m}$ long. Despite the different parameter selection all the three designs show a coupling efficiency larger than 0.75 and back-reflections smaller than $-20 \mathrm{~dB}$.

While coupling efficiency and back-reflections have been threated so far as deterministic quantities, they are unavoidably subject to some uncertainty in real devices due to fabrication tolerance. The investigation of their stochastic properties is therefore fundamental. On the other hand, even limiting the investigation only to the reduced parameter space, the analysis of the stochastic behavior for each possible alternative design with a classical Monte Carlo approach would be unfeasible, requiring at least hundreds of thousands of electromagnetic simulations. As described in Sec. 2 we can tackle the problem computing a surrogate model for the quantities of interest (coupling efficiency and backreflection in this example) exploiting a polynomial chaos expansion technique. As a first analysis we consider coupling efficiency CE and back-reflections R at $\lambda=1550 \mathrm{~nm}$ and PCE coefficients are then frequency-independent. We assume for both shallow and deeply etched sections in the grating the same random width deviation $\delta$, normally distributed with zero mean and a standard deviation of $5 \mathrm{~nm}$. For the coupling efficiency, an order $\mathrm{P}=3$ is enough to provide a good fit. Since we have only one random variable $(\mathrm{N}=1), \mathrm{K}=8$ values for $\delta$ are sampled according to its distribution and 

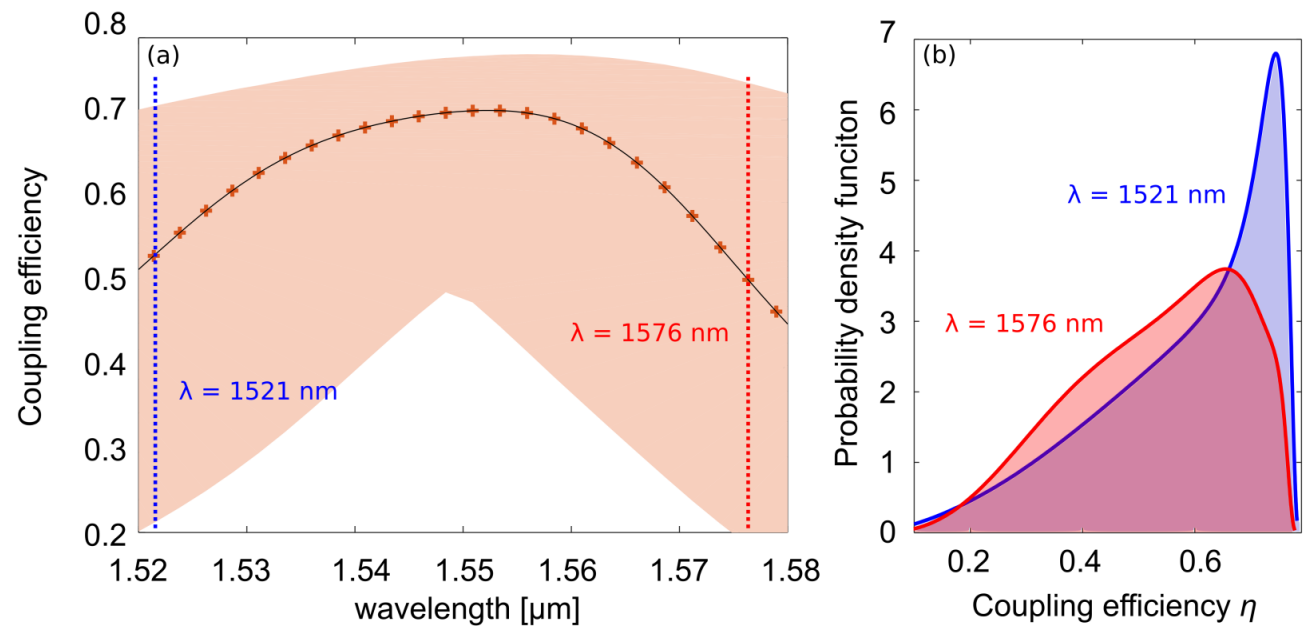

Figure 3: (a) Coupling efficiency spectrum for design 3. The shaded area is spanned by 40 Monte Carlo simulations. Combining vector-fitting and PCE the frequency-dependent surrogate model can be built for each of the 24 wavelength points used in the simulations. Crosses show the average coupling efficiency analytically derived from the model. Taking advantage of the vectorfitting capabilities the wavelength resolution can be increased without any new simulation. The probability density function of the coupling efficiency can be calculated at any wavelength sampling the surrogate model. Blue and red dashed line mark two wavelength at the extreme of the considered bandwidth $(1521 \mathrm{~nm}$ and $1576 \mathrm{~nm}$, respectively). The corresponding probability density functions are quite different and are shown in (b).

the corresponding designs are simulated with 2D-FDTD. The 8 simulations (vector $\mathbf{R}$ in Eq. (4)) are finally used to estimate the coefficients of the Hermite the four polynomial coefficients with a compressed sensing technique solving a basis pursuit denoise problem with the freely available spgl1 solver [13]. The computational time required to build the surrogate model (1) is negligible compared to simulation time. For the surrogate model of back-reflections, fifteenthorder Hermite polynomials are needed, with 30 initial simulations. The entire analysis is performed with a number of simulations orders of magnitude smaller than Monte Carlo, making possible its application to the large number of designs shown in Fig. 1(b,c).

Figure 2 shows as an example the probability density functions of coupling efficiency and back-reflections for designs 1 , 2 , and 3 . The probability density functions are obtained by sampling the surrogate models 5000 times (which only takes few seconds) and using a Gaussian kernel density estimator. Mean values are compute using Eq. (5). During the analysis, possible criteria to evaluate fabrication yield are also set requiring either $\mathrm{CE}>0.7$ or $\mathrm{R}<-20 \mathrm{~dB}$. The number of devices respecting the criteria (yield) are computed integrating the corresponding section of the probability density function. The probability density functions of coupling efficiency (Fig. $2(\mathrm{a}, \mathrm{c})$ ) are right-bounded by the value obtained without considering uncertainty (see Tab. 1). Design 1 shows a longer tail towards lower values of CE compared to design 2, reducing the probability to obtain a high coupling efficiency and consequently the yield (64\% instead of $78 \%)$. Design 3 has the lowest ideal and mean coupling efficiency $(0.75$ and 0.68 , respectively) and the lowest yield $(60 \%)$. Probability density functions for back-reflections are remarkably different for the three designs. Without uncertainty design 2 has substantially smaller back-reflections compared to designs 1 and 3 (Tab. 1) but they quickly grow when width variations are introduced (longer tail of the function). In contrast, the minimum back-reflection achievable by design 1 and 3 is much higher but its variability is considerably smaller, as demonstrated by the narrower probability density functions. For designs 1 and 2 back-reflections do not exceed $-10 \mathrm{~dB}$ with the considered uncertainty. The worstcase scenario for design 3 shows higher back-reflections (about $-7 \mathrm{~dB}$ ) but a slightly lower average $(-19 \mathrm{~dB})$. With the chosen criteria design 3 has also the largest expected yield (46\%).

As described in Sec. 2, PCE can be combined with frequency-domain macromodeling (vector fitting) to efficiently build stochastic surrogate models of frequency-dependent quantities. We consider here the same uncertainty described in the previous paragraph and analyze its effect on the entire coupling efficiency spectrum, computed on 24 wavelength points from $1520 \mathrm{~nm}$ to $1580 \mathrm{~nm}$. The quantity of interest $\operatorname{CE}(\lambda, \delta)$ depends now on wavelength and on one stochastic variable (width deviation). For each of the $\mathrm{K}=40$ initial simulations we model $\mathrm{CE}$ dependence on wavelength through vector 
(a)

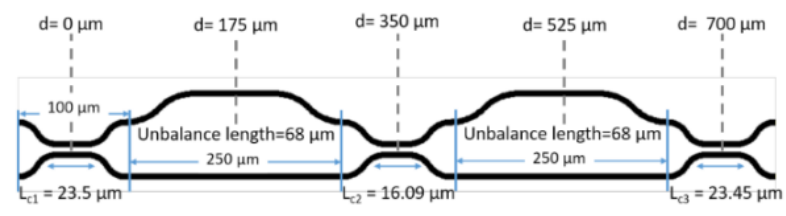

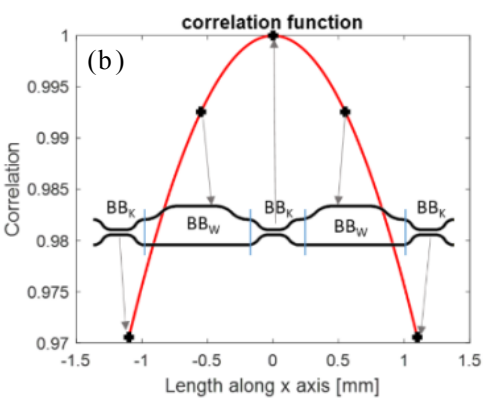

Figure 4: (a) Schematic of the second-order Mach-Zehnder filter. (b) Variation of the correlation function along the x-axis. Each cross mark represents the value of correlation of the respective building block of the circuit.

fitting with 6 poles and then apply PCE on the resulting matrices using polynomial order P $=6$ to obtain the final PCEVF surrogate model. Results for design 3 are shown in Fig. 3(a). The 40 simulations used to build the model are included in the shaded area. A much larger variability can be seen at the edges of the spectrum compared to the grating central wavelength $1550 \mathrm{~nm}$. Crosses mark the average coupling efficiency at each of the 24 wavelength points calculated with Eq. (5). Besides making PCE more efficient, the use of vector fitting to model the wavelength dependence of CE allows to improve wavelength resolution without performing additional simulations, computing stochastic moments at any wavelength within the considered range. As an example, the solid black line in Fig. 3(a) shows the average value of CE computed on 250 points while Fig. 3(b) shows the CE probability density function at $\lambda=1521 \mathrm{~nm}$ (blue curve) and $\lambda=$ $1576 \mathrm{~nm}$ (red curve). The two functions well highlight how the behavior of coupling efficiency under width variability strongly changes with wavelength.

\section{ANALYSIS OF PHOTONIC CIRCUITS WITH CORRELATED VARIABLES}

While for compact photonic devices the assumption of perfectly correlated variables can be a rather accurate description of the real variability (in the previous section we consider the same width deviation $\delta$ for the entire grating coupler) this condition might not be completely fulfilled when large circuits are considered. A finite correlation between uncertain parameters basically means that devices placed adjacent to one another on a chip tend to exhibit less mismatch variation than devices that are separated by a large distance. In literature, some techniques are available for statistical analysis in photonics including correlation that are based on either layout-dependent Monte Carlo [7] or arbitrary polynomial chaos $[10,11]$. The latter requires calculating basis functions to build the model (1) using complex algorithms. In this section, we will instead demonstrate the use of polynomial chaos expansion with correlated samples, for which Karhunen-Loeve (KL) transform is introduced.

As an example we analyze a second-order unbalanced Mach-Zehnder filter. Circuit performance is evaluated in presence of correlated manufacturing uncertainties in the design parameters that are the widths of the waveguides and the couplers' gaps. The accuracy of the results is compared against Monte Carlo. The considered Mach-Zehnder filter has a nominal 3-dB bandwidth of $\mathrm{BW}_{0}=378 \mathrm{GHz}$, and the corresponding coupling coefficients for the three directional couplers are $\mathrm{K}_{1}=\mathrm{K}_{3}=0.865$ and $\mathrm{K}_{2}=0.532$, with in-band isolation larger than $20 \mathrm{~dB}$. For the directional coupler building block, the considered nominal gap is $\mathrm{g}_{\mathrm{i}}=0.3 \mu \mathrm{m}$, leading to coupling lengths of $\mathrm{L}_{\mathrm{c} 1}=\mathrm{L}_{\mathrm{c} 3}=23.45 \mu \mathrm{m}$, and $\mathrm{L}_{\mathrm{c} 3}=16.09 \mu \mathrm{m}$. For the waveguide building blocks, the nominal waveguide width is $\mathrm{W}_{\mathrm{i}}=408 \mathrm{~nm}$ and its thickness is $220 \mathrm{~nm}$, corresponding to an effective index and group index of about 2.23 and 4.402, respectively. Both have the same unbalance lengths of $68 \mu \mathrm{m}$, corresponding to a free spectral range of FSR $=1 \mathrm{THz}$. The starting point to introduce correlation between building blocks is to obtain the value of the correlation coefficient for each circuit's component. This can be done using the correlated surface function, which is given as [4]

$$
g(x, y)=e^{-\frac{\left(x^{2}+y^{2}\right)}{2 l^{2}}}
$$

where $l$ is the correlation length, and $x$ and $y$ are the coordinate of the circuit's component on $\mathrm{x}-\mathrm{y}$ layout plane. The layout of the second-order unbalanced Mach-Zehnder filter is shown in Fig. 4(a). The vertical dashed line represents the 


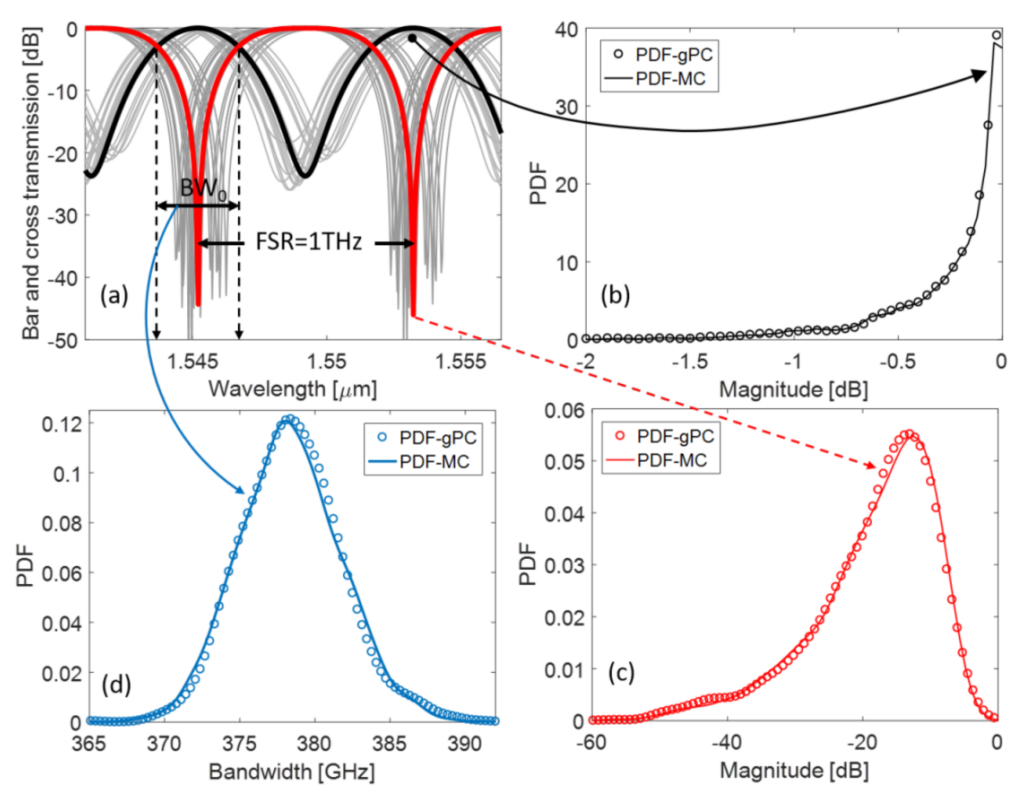

Figure 4: (a) MC analysis of circuit. PDF of the intensity transfer function at the (b) bar (black) and (c) cross (red) ports of the filter at the central wavelength, respectively. (d) PDF of the 3-dB bandwidth at the bar port of the filter. Full lines and circles are the PDFs computed using MC and gPC techniques, respectively. For both methods, 104 samples are used.

distance between the building blocks (i.e., directional coupler and waveguide) used to build the circuit. Based on the layout of the Mach-Zehnder filter, the correlation function is obtained considering a correlation length of $4.5 \mathrm{~mm}$ [19], as shown in Fig. 4(b). Without loss of generality, we assume that all building blocks are aligned in the x-axis, and therefore the value of $\mathrm{y}$ is fixed. Using the above approach, a correlation matrix of size $\mathrm{N} \times \mathrm{N}$ is generated, where $\mathrm{N}$ is the number of building blocks. For each building block, we consider one uncertain parameter and therefore the total number of uncertain parameters is $\mathrm{N}=5$. Once the number of uncertain parameters and the correlation matrix are known, the correlated samples are generated from uncorrelated samples using KL transform, which is based on the spectral decomposition of the correlation matrix. For the stochastic analysis, the width of the coupler's gap and the waveguide width of the stages are considered as normally distributed random variables with standard deviation $\sigma_{\mathrm{W}}=1 \mathrm{~nm}$. The PCE of the circuit transfer function is built using the same procedure described in Sec. 2. Instead of using the correlated samples (generated by means of KL transform) for the linear regression, we use the uncorrelated ones (before transformation) to exploit the orthogonality of the polynomial basis (Hermite polynomial) with respect to the distribution of the uncorrelated parameters. Using correlated samples for the linear regression would instead imply the calculation of a suitable set of new orthogonal polynomial basis functions [10,11]. It should be noted that more general and viable approaches were recently proposed to handle non-Gaussian correlated uncertain parameters $[12,20]$. Nevertheless, using the described procedure to build the PCE approximation works well for correlated Gaussian parameters. After obtaining the PCE model, the circuit's statistical behavior is inexpensively computed as described in the previous sections. Figure 5 shows as an example the probability density function of the amplitude of the filter transmission at the bar and cross ports and the distribution of the filter bandwidth.

\section{CONCLUSIONS}

In this work we have shown how polynomial chaos expansion can be fruitfully exploited to efficiently investigate the stochastic properties of photonic devices and circuits subject to fabrication uncertainty. The methodology was applied to both a problem with a single random variable and to a problem with multiple correlated variables. In both cases a rich description of the stochastic properties of the devices was retrieved using a very limited amount of time expensive simulations. This makes the approach attractive for the investigation of large multi-parameter design space and machineassisted design approaches requiring the analysis of a large number of possible alternative design solutions. 


\section{REFERENCES}

[1] P. Cheben, R. Halir, J. H. Schmid, H. A. Atwater, and D. R. Smith, "Subwavelength integrated photonics," Nature, vol. 560, no. 7720, pp. 565-572, Aug. 2018.

[2] L. F. Frellsen, Y. Ding, O. Sigmund, and L. H. Frandsen, "Topology optimized mode multiplexing in silicon-oninsulator photonic wire waveguides," Optics Express, vol. 24, no. 15, p. 16866, Jul. 2016.

[3] J. S. Jensen and O. Sigmund, "Topology optimization for nano-photonics," Laser \& Photonics Reviews, vol. 5, no. 2, pp. 308-321, Mar. 2011.

[4] A. Y. Piggott, J. Lu, K. G. Lagoudakis, J. Petykiewicz, T. M. Babinec, and J. Vučković, "Inverse design and demonstration of a compact and broadband on-chip wavelength demultiplexer," Nature Photonics, vol. 9, no. 6, pp. 374377, Jun. 2015.

[5] J. Peurifoy et al., "Nanophotonic particle simulation and inverse design using artificial neural networks," Science Advances, vol. 4, no. 6, p. eaar4206, Jun. 2018.

[6] D. Melati, Y. Grinberg, S. Janz, P. Cheben, J. H. Schmid, A. Sánchez-Postigo, D.-X. Xu "Mapping the global design space of integrated photonic components using machine learning pattern recognition", OSF Preprints. Nov. 2018. [Online]. Available: doi.org/10.31219/osf.io/xmnjs.

[7] Z. Lu et al., "Performance prediction for silicon photonics integrated circuits with layout-dependent correlated manufacturing variability," Optics Express, vol. 25, no. 9, p. 9712, May 2017.

[8] A. Waqas, D. Melati, and A. Melloni, "Sensitivity Analysis and Uncertainty Mitigation of Photonic Integrated Circuits," Journal of Lightwave Technology, vol. 35, no. 17, pp. 3713-3721, 2017.

[9] A. Waqas, D. Melati, P. Manfredi, and A. Melloni, "Stochastic process design kits for photonic circuits based on polynomial chaos augmented macro-modelling," Opt. Express, vol. 26, no. 5, pp. 5894-5907, Mar. 2018.

[10] Y. Xing, D. Spina, A. Li, T. Dhaene, and W. Bogaerts, "Stochastic collocation for device-level variability analysis in integrated photonics," Photonics Research, vol. 4, no. 2, p. 93, Apr. 2016.

[11] T.-W. Weng, Z. Zhang, Z. Su, Y. Marzouk, A. Melloni, and L. Daniel, "Uncertainty quantification of silicon photonic devices with correlated and non-Gaussian random parameters," Optics Express, vol. 23, no. 4, Feb. 2015.

[12] C. Cui and Z. Zhang, "Stochastic Collocation with Non-Gaussian Correlated Process Variations: Theory, Algorithms and Applications," IEEE Transactions on Components, Packaging and Manufacturing Technology, pp. 1-1, 2018.

[13] T.-W. Weng, D. Melati, A. Melloni, and L. Daniel, "Stochastic simulation and robust design optimization of integrated photonic filters," Nanophotonics, vol. 6, no. 1, pp. 299-308, Jan. 2017.

[14] D. Melati, A. Waqas, D.-X. Xu, and A. Melloni, "Genetic algorithm and polynomial chaos modelling for performance optimization of photonic circuits under manufacturing variability," in Optical Fiber Communication Conference, 2018, p. M3I.4.

[15] M. Eldred, "Recent Advances in Non-Intrusive Polynomial Chaos and Stochastic Collocation Methods for Uncertainty Analysis and Design," 2009.

[16] D. Spina, F. Ferranti, T. Dhaene, L. Knockaert, G. Antonini, and D. Vande Ginste, "Variability Analysis of Multiport Systems Via Polynomial-Chaos Expansion," IEEE Transactions on Microwave Theory and Techniques, vol. 60, no. 8, pp. 2329-2338, Aug. 2012.

[17] T. Watanabe, M. Ayata, U. Koch, Y. Fedoryshyn, and J. Leuthold, "Perpendicular Grating Coupler Based on a Blazed Antiback-Reflection Structure," Journal of Lightwave Technology, vol. 35, no. 21, pp. 4663-4669, Nov. 2017.

[18] L. Zavargo-Peche, A. Ortega-Monux, J. G. Wanguemert-Perez, I. Molina-Fernandez, "Fourier based combined techniques to design novel sub-wavelength optical integrated devices," Prog. Electromagn. Res. 123, 447-465, 2012.

[19] Yisu Yang et al., Phase coherence length in silicon photonic platform. Optics express, 23(13):16890-16902, 2015.

[20] C. Cui et al., "Uncertainty quantification of electronic and photonic ICs with non-Gaussian correlated process variations," arXiv: $1807.01778 \mathrm{v} 1$ 